This item was submitted to Loughborough's Institutional Repository (https://dspace.lboro.ac.uk/) by the author and is made available under the following Creative Commons Licence conditions.

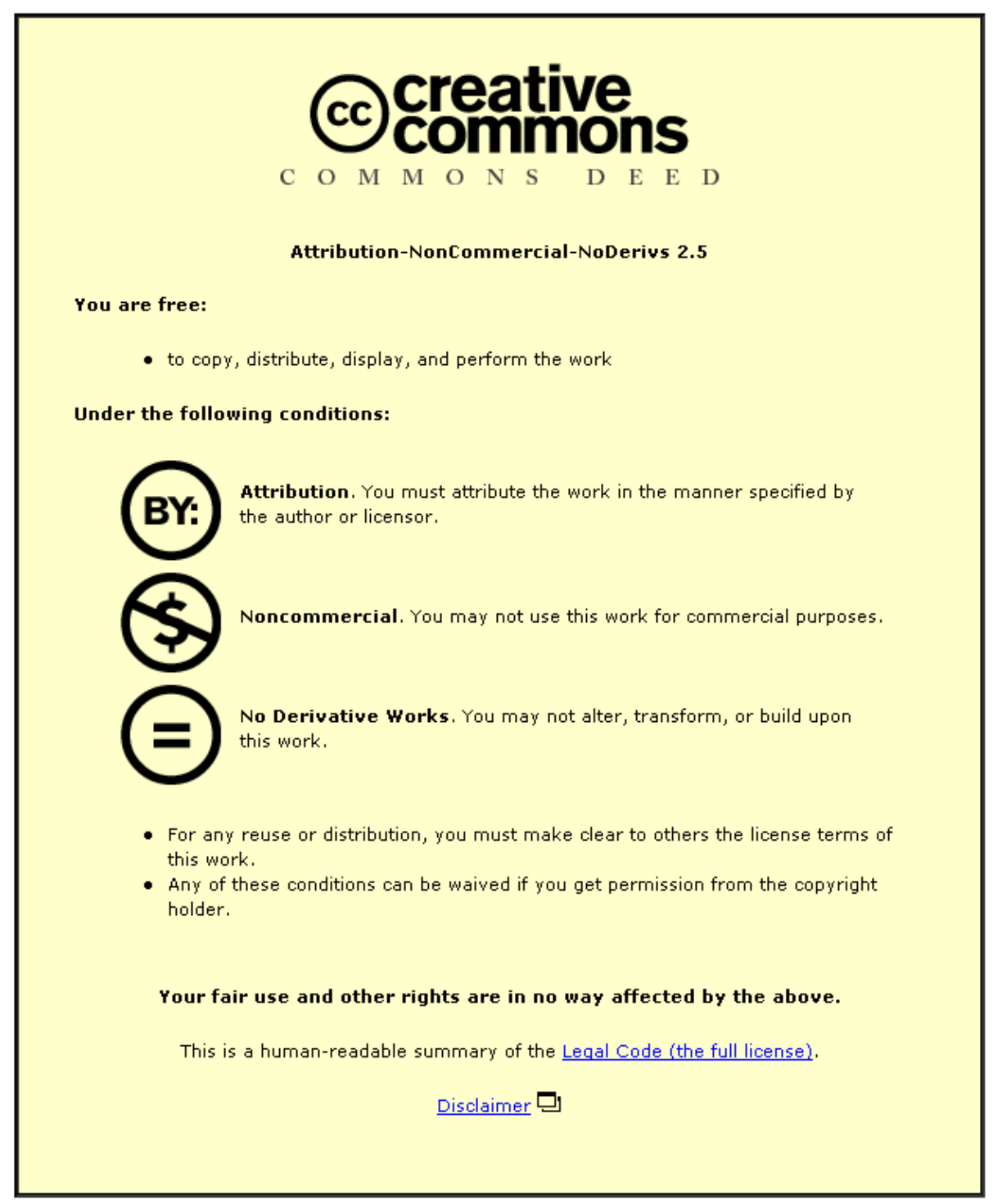

For the full text of this licence, please go to: http://creativecommons.org/licenses/by-nc-nd/2.5/ 


\title{
Signatures of chaoticlike and nonchaoticlike behavior in a nonlinear quantum oscillator through photon detection
}

\author{
M. J. Everitt, ${ }^{1,2, *}$ T. D. Clark, ${ }^{2,3}$ P. B. Stiffell, ${ }^{2}$ J. F. Ralph, ${ }^{3}$ A. R. Bulsara, ${ }^{4}$ and C. J. Harland ${ }^{2}$ \\ ${ }^{1}$ The British University in Egypt, El Sherouk City, Misr Ismalia Desert Road, Postal No. 11837 P.O. Box 43, Egypt \\ ${ }^{2}$ Centre for Physical Electronics and Quantum Technology, School of Science and Technology, University of Sussex, Falmer, Brighton, \\ BN1 9QT, United Kingdom \\ ${ }^{3}$ Department of Electrical and Electronic Engineering, Liverpool University, Brownlow Hill, Liverpool, L69 3GJ, United Kingdom \\ ${ }^{4}$ Space and Naval Warfare Systems Center, Code 2363, 53560 Hull Street, San Diego, California 92152-5001, USA
}

(Received 24 March 2005; published 19 December 2005)

\begin{abstract}
The driven nonlinear Duffing oscillator is a very good, and standard, example of a quantum mechanical system from which classical-like orbits can be recovered from unravelings of the master equation. In order to generate such trajectories in the phase space of this oscillator, in this paper we use the quantum jump unraveling together with a suitable application of the correspondence principle. We analyze the measured readout by considering the power spectra of photon counts produced by the quantum jumps. Here we show that localization of the wave packet from the measurement of the oscillator by the photon detector produces a concomitant structure in the power spectra of the measured output. Furthermore, we demonstrate that this spectral analysis can be used to distinguish between different modes of the underlying dynamics of the oscillator.
\end{abstract}

DOI: 10.1103/PhysRevE.72.066209 PACS number(s): 05.45.Mt, 03.65.Ta, 03.65.Sq, 42.50.Lc

\section{INTRODUCTION}

There is currently intense interest being shown in the possible application of quantum devices to fields such as computing and information processing [1]. The goal is to construct machinery that operates manifestly at the quantum level. In any successful development of such technology the role of measurement in quantum systems will be of central, indeed crucial, importance (see for example Ref. [2]). In order to extend our understanding of this problem we have recently investigated the coupling together of quantum systems that, to a good approximation, appear classical (via the correspondence limit) but whose underlying behavior is strictly quantum mechanical [3]. In this work we followed the evolution of two coupled, and identical, quantized Duffing oscillators as our example system. We utilized two unravelings of the master equation to describe this system: quantum state diffusion and quantum jumps which correspond, respectively, to unit-efficiency heterodyne measurement (or ambiquadrature homodyne detection) and photon detection [4]. We demonstrated that the entanglement that exists between the two oscillators depends on the nature of their dynamics. Explicitly, we showed that while the dynamics was chaoticlike the entanglement between the oscillators remained high; conversely, if the two oscillators entrained into a periodic orbit the degree of entanglement became very small.

With this background we subsequently became interested in acquiring a detailed understanding of experimental readouts of quantum chaoticlike systems. In this paper we have chosen to explore the subject through the quantum jump unraveling of the master equation [4-6]. Here, the measured output is easily identified, namely, a click or no click in the

\footnotetext{
*Electronic address: m.j.everitt@physics.org
}

photon detector. However, this measurement process is unique in the fact that it possesses no classical analog. Indeed, this is the case even when the system under consideration may appear to be evolving along a classical trajectory. Interestingly, despite the fact that the photon detector has no classical analog, it is the very presence of this as a source of decoherence that is responsible for recovering classical-like orbits in the $(\langle q\rangle,\langle p\rangle)$ phase plane (despite the fact that we measure neither $q$ nor $p$ ). The subject of recovering such chaoticlike dynamics from unravelings of the master equation has been studied in depth in the literature [7-11] and a detailed discussion is beyond the scope of this paper. However, we note that recently in Ref. [12] resonances have been observed in a model of a nonlinear nanomechanical resonator that are absent in the corresponding classical model. In this present work we have chosen to scale the oscillator so that we recover orbits similar to those generated from a classical analysis.

\section{BACKGROUND}

In this work we study the output resulting from the measurement of quantum objects where the measurement device generates decoherence effects. In this limit the system exhibits dynamical behavior in terms of its expectation values very much like those observed in its classical counterpart. In this work we investigate the region of parameter space under which the classical system exhibits chaotic motion. Of the many models that could be used we have chosen the quantum jump approach [4-6]. We note that this is only one of several possible unravelings of the master equation that correspond to the continuous measurement of the quantum object considered. Our motivation for using this approach is that the recorded output of the measurement is completely transparent, i.e., the photon counter either registers a photon or it does not. 

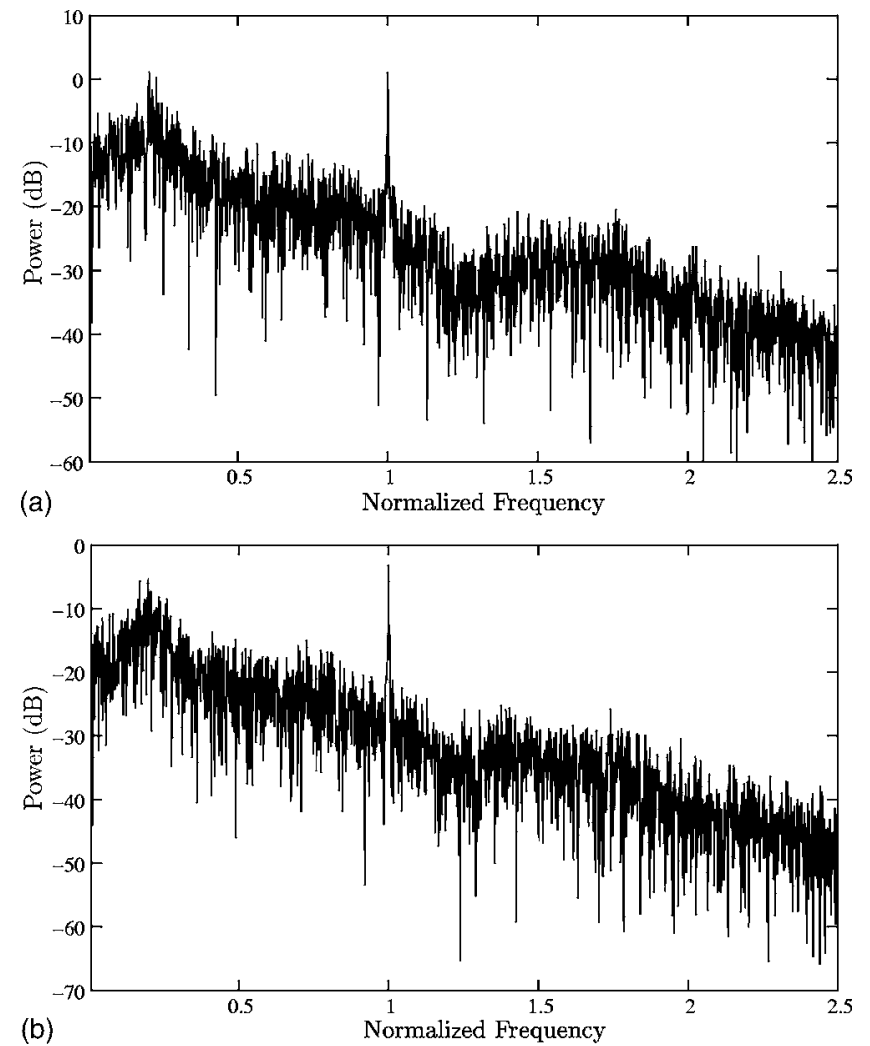

FIG. 1. Power spectra of the position $x$ for the classical Duffing oscillator and $\langle q\rangle$ for the quantum Duffing oscillator $\beta=0.1$. The frequency is normalized to the drive frequency of the oscillator.

In the quantum jump unraveling of the master equation the evolution of the (pure) state vector $|\psi\rangle$ for an open quantum system is given by the stochastic Itô increment equation

$$
\begin{aligned}
|d \psi\rangle= & -\frac{i}{\hbar} H|\psi\rangle d t-\frac{1}{2} \sum_{j}\left(L_{j}^{\dagger} L_{j}-\left\langle L_{j}^{\dagger} L_{j}\right\rangle\right)|\psi\rangle d t \\
& +\sum_{j}\left(\frac{L_{j}}{\sqrt{\left\langle L_{j}^{\dagger} L_{j}\right\rangle}}-1\right)|\psi\rangle d N_{j}
\end{aligned}
$$

where $H$ is the Hamiltonian, $L_{i}$ are the Lindblad operators that represent coupling to the environmental degrees of freedom, $d t$ is the time increment, and $d N_{j}$ is a Poissonian noise process such that $d N_{j} d N_{k}=\delta_{j k} d N_{j}, d N_{j} d t=0$, and $\overline{d N_{j}}$ $=\left\langle L_{j}^{\dagger} L_{j}\right\rangle d t$. These latter conditions imply that jumps occur randomly at a rate that is determined by $\left\langle L_{j}^{\dagger} L_{j}\right\rangle$. We will find that this is very important when explaining the results presented later in this paper. For an excellent discussion of quantum trajectories interpreted as a realistic model of a system that is being continuously monitored, see Ref. [4]. For an interesting and more general discussion on the emergence of classical-like behavior from quantum systems, see Refs. $[13,14]$.

The Hamiltonian for our, standard, example system of the Duffing oscillator is given by
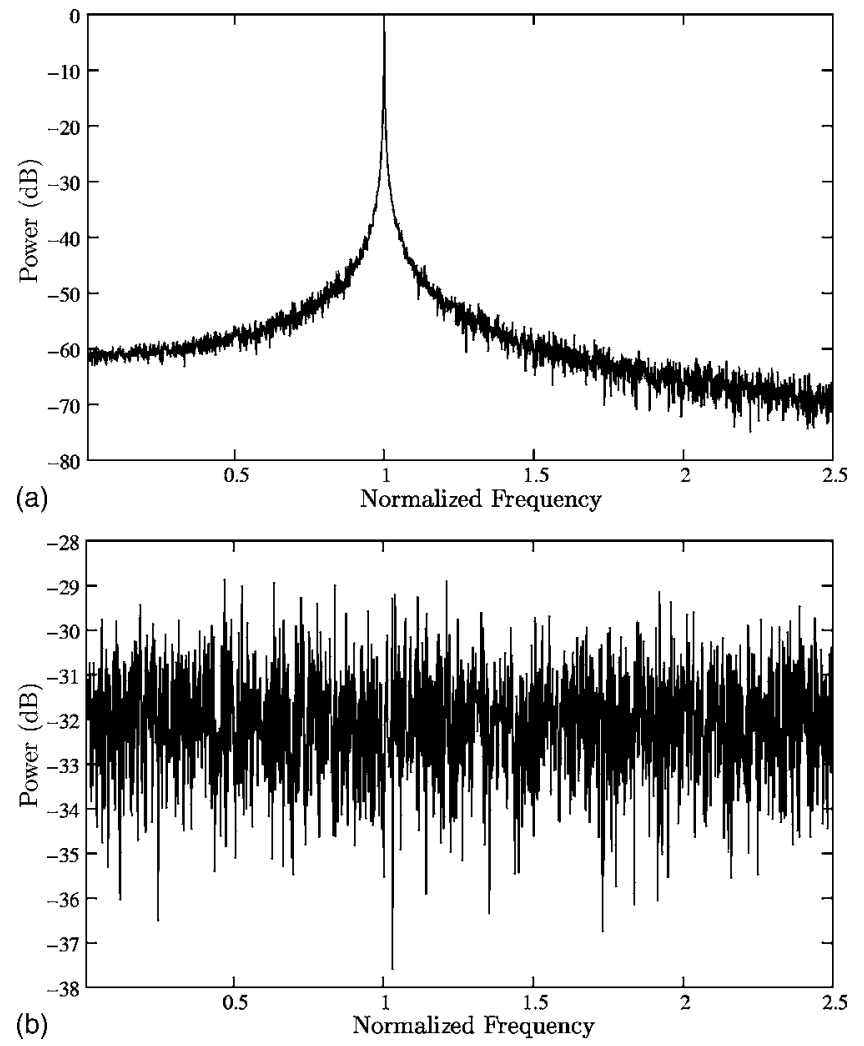

FIG. 2. Power spectra of the position $\langle q\rangle$ and photons counted $\mathcal{N}(t)$ for the quantum simple harmonic oscillator in a steady state. Here the frequency is normalized to the drive frequency of the oscillator.

$$
H=\frac{1}{2} p^{2}+\frac{\beta^{2}}{4} q^{4}-\frac{1}{2} q^{2}+\frac{g}{\beta} \cos (t) q+\frac{\Gamma}{2}(q p+p q)
$$

where $q$ and $p$ are the canonically conjugate position and momentum operators for the oscillator. In this example we have only one Lindblad operator which is $L=\sqrt{2 \Gamma} a$, where $a$ is the oscillator annihilation (lowering) operator, $g$ is the drive amplitude, and $\Gamma=0.125$ quantifies the damping.

In order to apply the correspondence principal to this system, and recover classical-like dynamics, we have introduced

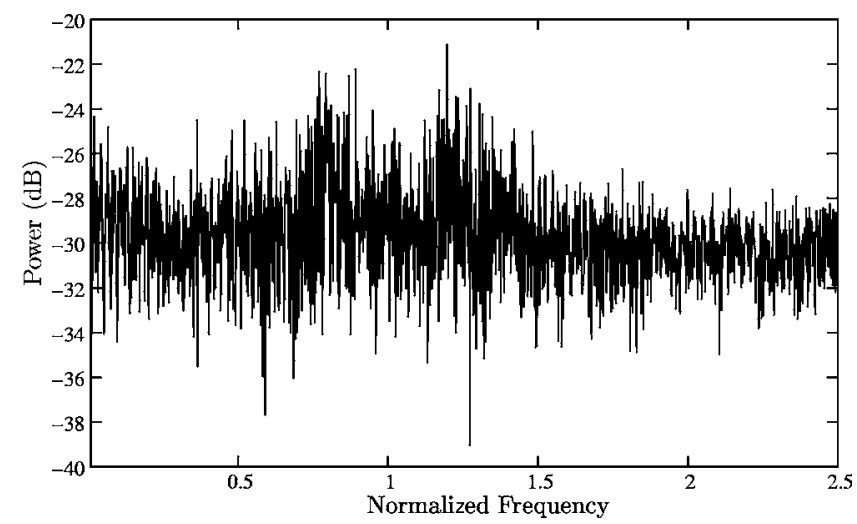

FIG. 3. Power spectrum of the measured quantum jumps $\mathcal{N}(t)$ for the Duffing oscillator of Fig. 4. 




(a)

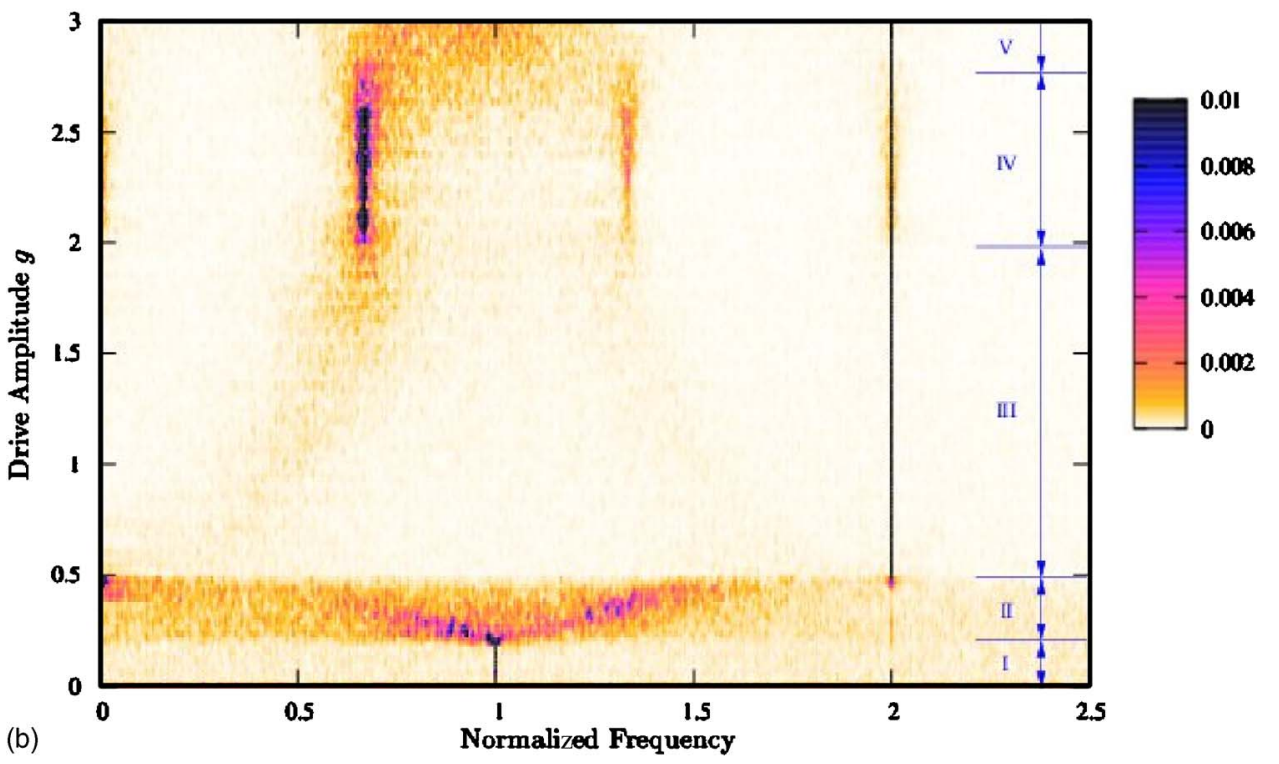

FIG. 4. (Color online) Power spectra of (a) $\langle q\rangle$ and (b) the measured quantum jumps as a function of drive amplitude. in Eq. (2) the parameter $\beta$. For this Hamiltonian it has two interpretations that are mathematically equivalent. First, it can be considered to scale $\hbar$ itself, or, alternatively we can simply view $\beta$ as scaling the Hamiltonian, leaving $\hbar$ fixed, so that the relative motion of the expectation values of the observables becomes large compared with the minimum area $(\hbar / 2)$ in the phase space. In either case, the system behaves more classically as $\beta$ tends to zero from its maximum value of 1 . In this work we have chosen to set $\beta=0.1$.

\section{RESULTS}

Let us now consider the specific example of a Duffing oscillator with a drive amplitude $g=0.3$. This parameter, together with all those already specified, form the classic example used to demonstrate that chaoticlike behavior can be recovered for open quantum systems by using unravelings of the master equation $[3,7,9,10]$. In Fig. 1 we compare the power spectra of the classical position coordinate with that of $\langle q\rangle$. Here noise has been added to the classical system so as to mimic the level of quantum noise that is present in the stochastic elements of our chosen unraveling of the master equation and we have solved for a realization of the Langevin equation. As can be seen, for this value of $\beta$ there is a very good match between these two results. Moreover, both display power spectra that are typical for oscillators in chaotic orbits.

However, it is not position that is the measured output in this model, but the quantum jumps recorded, as a function $\mathcal{N}(t)$ of time in the photon detector. As stated above, these jumps occur randomly at a rate that is determined by $\left\langle L_{j}^{\dagger} L_{j}\right\rangle$ which, for this example, is $2 \Gamma\langle n\rangle$. Hence, the probability of making a jump is proportional to the number of photons in the state of the system at any one time.

We now consider a special case that occurs frequently in the classical limit, namely, where $|\psi\rangle$ localizes approximately to a coherent (Gaussian) state. It is apparent that for such a state the chance of observing a jump is proportional to the square of the distance in $(\langle q\rangle,\langle p\rangle)$ of the state from the ori- 

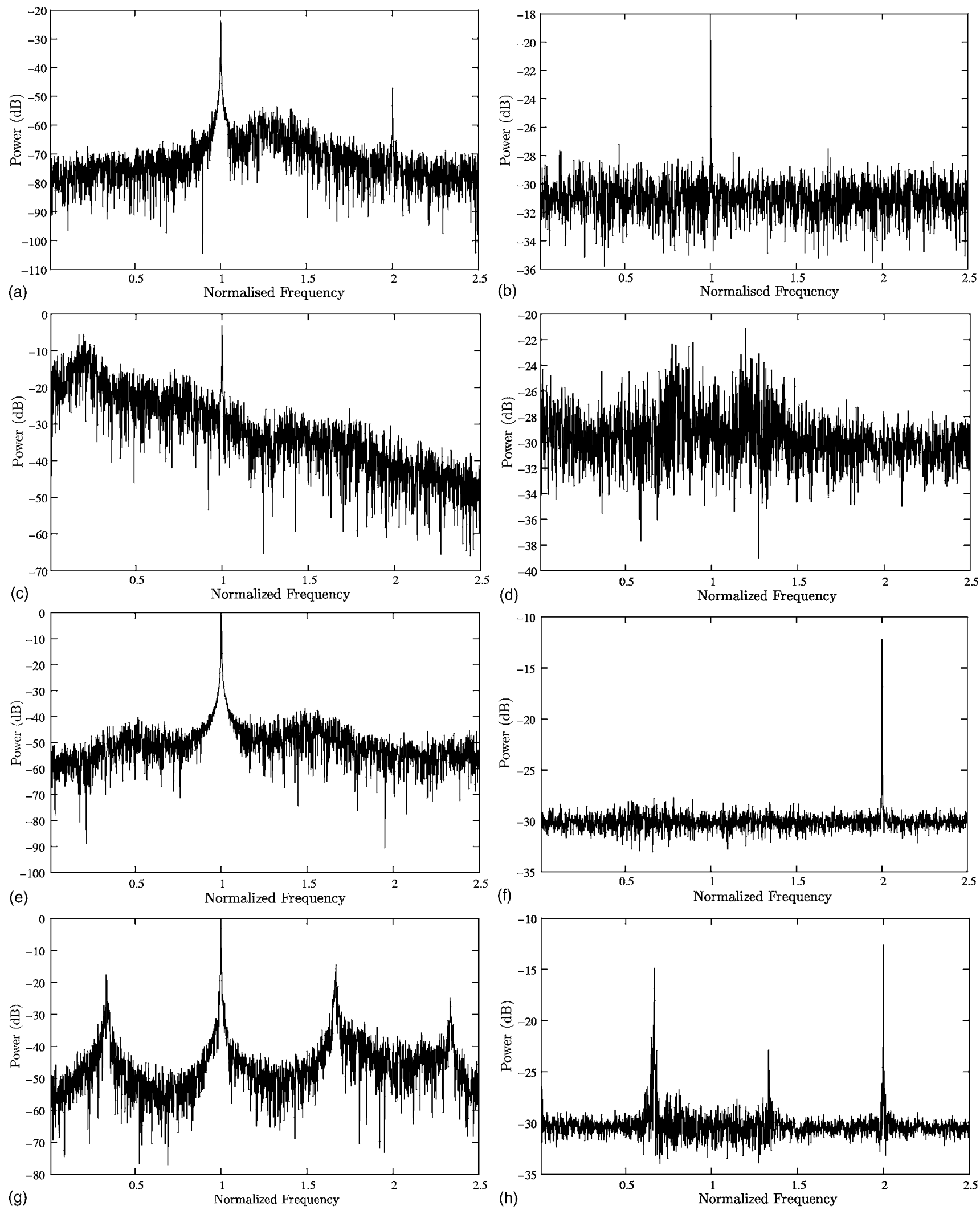

FIG. 5. Example power spectra for four different drive amplitudes corresponding to the regions I to IV as marked in the power spectrum of Fig. 4. 
gin. In order to illustrate the implications of this, let us consider a driven simple harmonic oscillator. The Hamiltonian is

$$
H_{s}=\frac{1}{2} p^{2}+\frac{1}{2} q^{2}+\frac{g}{\beta} \cos (t) q
$$

and we note that in this special case the only effect of $\beta=0.1$ is to scale the amplitude of the drive (again we set $g=0.3$ ). We now solve Eq. (1) using this Hamiltonian and allow the system to settle into a steady state. Then, as the phase portrait for this system simply describes a circle centered about $(0,0)$ we would expect the power spectra of photons counted to be the same as those for white noise. Indeed, this is clearly seen in Fig. 2 where we show the power spectrum for both (a) the position operator and (b) the measured quantum jumps.

For more complicated orbits, such as those exhibited by the Duffing oscillator, we would expect to see some evidence of the underlying dynamical behavior. Hence, localization of $|\psi\rangle$ from the measurement of the Duffing oscillator through the photon detector forms a concomitant structure in the power spectrum of the measured output. In Fig. 3 we show, for comparison with Fig. 1(b), such a power spectrum.

As we can see from Fig. 3 the power spectrum for this chaotic mode of operation reveals some structure. However, it is not clear from this picture alone how we might relate this result to that shown in Fig. 1(b). It is therefore reasonable to ask if this result does indeed tell us anything about the underlying dynamics of the oscillator. We have addressed this point by computing the power spectrum of both $\langle q\rangle$ and $\mathcal{N}(t)$ for drive amplitudes in the range $0<g \leqslant 3$, the results of which are presented in Fig. 4.

Although the functional forms of these power spectra obviously differ, they do clearly exhibit changes in behavior that are coincident in the drive amplitudes of both figures. These are identified as intervals in $g$ labeled I, II,... in Fig. 4.

To help clarify Fig. 4 we provide in Fig. 5 explicit power spectra of both $\langle q\rangle$ and the quantum jumps $\mathcal{N}(t)$ for regions I-IV. As expected for region I in Figs. 4 and 5(a,b) we see a strong resonance at the frequency of the drive. The broadband behavior characteristic of the chaotic phenomena associated with region II is evident in Fig. 5(c) and a concomitant, although different, structure in the power spectrum [Fig. 5(d)] of the detected photons. In region III of Fig. 4 we again return to a periodic orbit. In Fig. 5(e), the power spectrum for $\langle q\rangle$ exhibits a peak at the drive frequency; however, the power spectrum [Fig. 5(f)] of $\mathcal{N}(t)$ peaks at twice this frequency. The lack of coincidence between these two figures will be explained fully in the following text. Finally in Figs. $5(\mathrm{~g})$ and $5(\mathrm{~h})$ we see the power spectra of the quasiperiodic dynamics of region IV; again the discrepancy between these two figures is discussed below.

The mechanism through which the detection of photons can yield significant information about the underlying dynamics of the system can be understood by looking at the phase portraits of $\langle q\rangle$ and $\langle p\rangle$ associated with the regions I-IV of Fig. 4 for those values of drive used in Fig. 5. These are shown in Fig. 6.
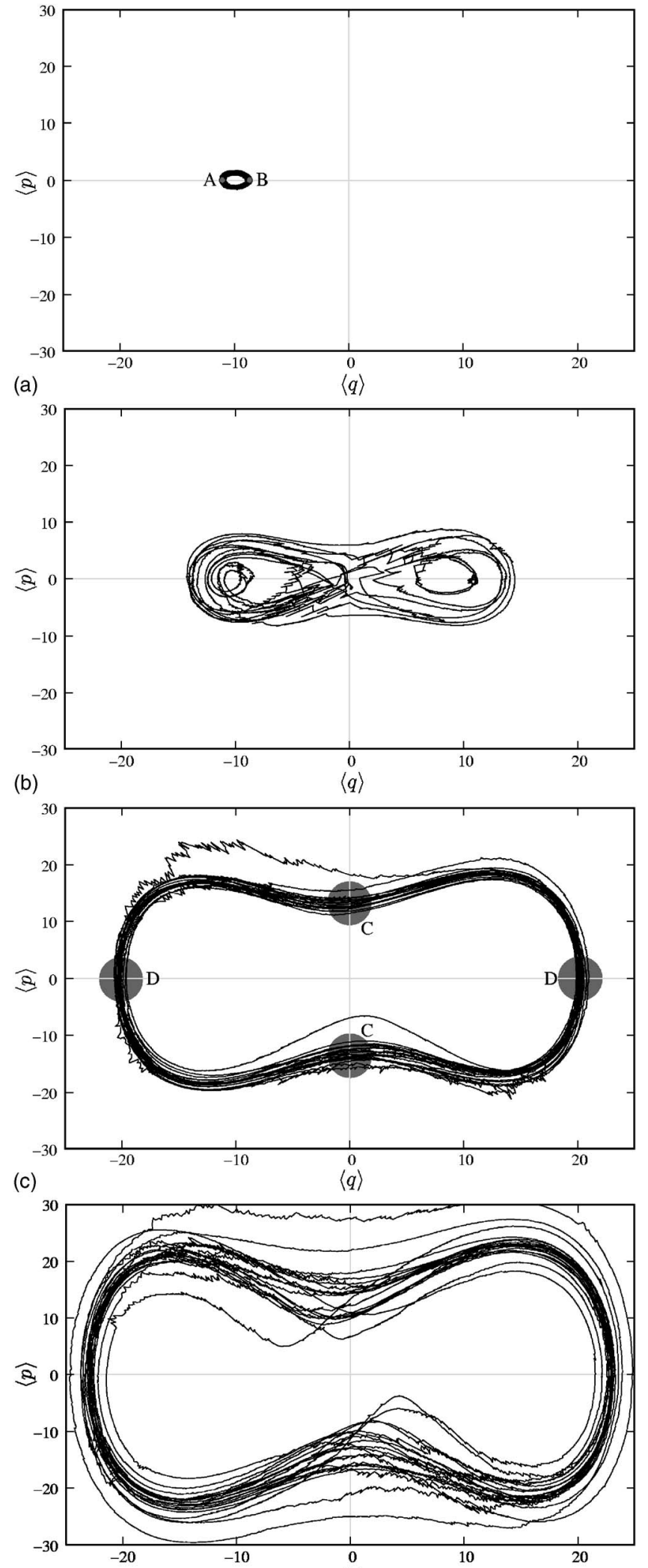

(d)

$\langle q\rangle$

FIG. 6. Example phase portraits for four different drive amplitudes corresponding to the regions I to IV as marked in the power spectrum of Fig. 4. 
For region I there is a strictly periodic response on both power spectra at the drive frequency of the oscillator. It can be seen from Fig. 6(a) that, because of the distance from the origin, the chance of there being a photon counted at point $A$ is higher than at point $B$. As this occurs at the same frequency as the oscillations of $\langle q\rangle$, we have direct agreement in the position of the resonance in each of the different spectra.

In region II, and as is clear from Fig. 6(b), the system is following a chaoticlike trajectory. Although the power spectra differ drastically in their structure, they do both exhibit broadband behavior that is characteristic of chaotic orbits.

As the drive amplitude is increased further, region III in Fig. 4 is accessed as the behavior observed in region II ceases. For this range of drive amplitudes the solution is again a stable periodic orbit as displayed in Fig. 6(c). However, this time, while the power spectrum of $\langle q\rangle$ exhibits a resonance at the drive frequency, that of $\mathcal{N}(t)$ appears at double this frequency. The explanation for this is simply that the probability of detecting a photon when the orbit is in a region of phase space near the origin, such as those marked $C$ in Fig. 6(c), is less than in those further away, as in the region $D$. This variation in probability occurs twice a period and therefore produces a resonance at double the drive frequency. An immediate corollary is that, by detecting a resonance at either of these different frequencies in the power spectra of $\mathcal{N}(t)$, we can determine whether the oscillator is in region I or III of Fig. 4. From our analysis in Ref. [3] it may, in some circumstances, be advantageous to place the system in a chaotic orbit. It is possible that this sort of analysis could be used to increase or decrease drive amplitude as part of a feedback and control element for quantum machinery.

Finally, the power spectrum of $\langle q\rangle$ in region IV of Fig. $6(c)$ is characteristic of quasiperiodic behavior. Using a similar argument to the one above, we can transfer these features onto the spectrum of $\mathcal{N}(t)$. If we compare this result with the, albeit noisy, phase portrait of Fig. 6(d) there is clear evidence of quasiperiodic behavior.
We have demonstrated, using the Duffing oscillator as our example system, that the different features exhibited in the power spectrum of the photon count can be associated with concomitant features in the power spectrum of the position operator (and vice versa). We note that for any given experimental system where there is a direct correspondence between the power spectrum of $\mathcal{N}$ and $\langle x\rangle$ the power spectrum of $\mathcal{N}$ provides us with the same amount of information about the underlying dynamics (e.g., chaotic, quasiperiodic, etc.) as the power spectrum of $\langle x\rangle$. We would like to emphasize that if this direct correspondence did not exist then we would not necessarily be able to make such an assertion. For example, this situation might occur for a system in which there was a high degree of symmetry in the $\langle x\rangle-\langle p\rangle$ phase portrait. However, such a detailed study is beyond the scope of this paper.

\section{CONCLUSION}

In this work we have shown that, via analysis of the power spectra of the photons detected in a quantum jump model of a Duffing oscillator, we can obtain signatures of the underlying dynamics of the oscillator. Again, we note that the decoherence associated with actually measuring these jumps is that which, through localization of the state vector, enables these classical-like orbits to become manifest. We have also demonstrated that the power spectra of the counted photons can be used to distinguish between different modes of operation of the oscillator. Hence, this or some form of time-frequency analysis could be used in the feedback and control of open quantum systems, a topic likely to be of interest in some of the emerging quantum technologies.

\section{ACKNOWLEDGMENTS}

The authors would like to thank T. P. Spiller and W. Munro for interesting and informative discussions. M.J.E. would also like to thank P. M. Birch for his helpful advice.
[1] M. Nielsen and I. Cheung, Quantum Computation and Information (Cambridge University Press, Cambridge, U.K., 2000).

[2] D. Shepelyansky, Phys. Scr., T 90, 112 (2001).

[3] M. Everitt, T. Clark, P. Stiffell, J. Ralph, A. Bulsara, and C. Harland, New J. Phys. 7, 64 (2005).

[4] H. M. Wiseman, Quantum Semiclassic. Opt. 8, 205 (1996).

[5] M. B. Plenio and P. L. Knight, Rev. Mod. Phys. 70, 101 (1998).

[6] G. C. Hegerfeldt, Phys. Rev. A 47, 449 (1993).

[7] I. Percival, Quantum State Diffusion (Cambridge University Press, Cambridge, U.K., 1998).

[8] S. Habib, K. Shizume, and W. H. Zurek, Phys. Rev. Lett. 80, 4361 (1998).
[9] T. A. Brun, N. Gisin, P. O’Mahony, and M. Gigo, Phys. Lett. A 229, 267 (1997).

[10] T. A. Brun, I. C. Percival, and R. Schack, J. Phys. A 29, 2077 (1996).

[11] T. P. Spiller and J. F. Ralph, Phys. Lett. A 194, 235 (1994).

[12] V. Peano and M. Thorwart, Phys. Rev. B 70, 235401 (2004).

[13] G. D. Giulini, E. Joos, C. Kiefer, J. Kupsch, I. Stamatescu, and H. Zeh, Decoherence and the Appearance of a Classical World in Quantum Theory (Springer-Verlag, Berlin, 1996).

[14] E. Joos, H. Zeh, C. Kiefer, G. D. Giulini, J. Kupsch, and I. Stamatescu, Decoherence and the Appearance of a Classical World in Quantum Theory, 2nd ed. (Springer-Verlag, Berlin, 2003). 